\title{
VARIAÇÕES DO CARBONO ORGÂNICO DISSOLVIDO E DE ATRIBUTOS FÍSICOS DO SOLO SOB DIFERENTES SISTEMAS DE USO DA TERRA NA AMAZÔNIA CENTRAL ${ }^{(1)}$
}

\author{
Jean Dalmo de Oliveira Marques ${ }^{(2)}$, Flávio Jesus Luizão( ${ }^{(3)}$, Wenceslau \\ Geraldes Teixeira $^{(4)} \&$ Sávio José Filgueiras Ferreira ${ }^{(3)}$
}

\begin{abstract}
RESUMO
O presente estudo teve como objetivo verificar a relação entre $\mathrm{C}$ orgânico dissolvido (COD) e atributos físicos do solo em diferentes sistemas de uso da terra na Amazônia central. Quantificaram-se as concentrações de COD sob floresta primária, em três posições topográficas, e em áreas de pastagem, sucessão secundária e sistema agroflorestal (SAF) até a profundidade de $2 \mathrm{~m}$. Instalaram-se extratores de solução do solo para coleta e análise do $\mathrm{C}$ da solução na fase orgânica durante um ciclo hidrológico. As concentrações médias de C orgânico dissolvido (COD) na solução do solo seguiram a ordem SAF ou sucessão secundária > floresta (platô) > pastagem (períodos seco e chuvoso-seco), exceto nos períodos chuvoso e seco-chuvoso, em que a pastagem iguala ou ultrapassa as concentrações de COD dos outros ambientes em determinadas profundidades. Os resultados demonstraram a capacidade do SAF e sucessão secundária em recuperar e, ou, disponibilizar o $\mathrm{C}$ na solução do solo, sendo formas de utilização do solo recomendadas para manter o $\mathrm{C}$ no ambiente terrestre. $\mathrm{O}$ COD apresentou variação temporal, espacial e em profundidade, decrescendo nesta última. A ação da estruturação do solo, representada pela agregação, $\mathrm{C}$ orgânico total (COT) e porosidade, exerce grande influência nas concentrações de COD nas camadas do solo e, aliada à matéria orgânica mais lábil do solo, determinam a
\end{abstract}

(1) Parte da Tese de Doutorado do primeiro autor, apresentada ao Curso de Pós Graduação em Ecologia, Instituto Nacional de Pesquisas da Amazônia - INPA. Bolsista CNPq. Recebido para publicação em 7 de outubro de 2010 e aprovado em 27 de janeiro de 2012.

(2) Professor do Instituto Federal de Educação, Ciência e Tecnologia do Amazonas - IFAM. Diretoria de Ensino Superior-DES. Av. Sete de Setembro 1975, Aleixo, CEP 69020-120 Manaus (AM).E-mails: jdomarques@hotmail.com, jeanmarques@ifam.edu.br

(3) Pesquisadores do Instituto Nacional de Pesquisas da Amazônia - INPA. Avenida Efigênio Sales 2239, Aleixo, CEP 69060-020 Manaus (AM). E-mails: fluizao@inpa.gov.br; savio@inpa.gov.br

(4) Pesquisador da Embrapa Solos. Rua Jardim Botânico 1024, Jardim Botânico, CEP 22460-000 Rio de Janeiro (RJ). E-mail: lau@ embrapa.brembrapa.br 


\begin{abstract}
manutenção do C na solução do solo. As concentrações de COD obtidas sob os diferentes ambientes estudados refletem a importância do manejo adequado do solo para a permanência do $\mathrm{C}$ no ecossistema do solo.
\end{abstract}

Termos de indexação: solução do solo, dinâmica do carbono no solo, cobertura vegetal, física do solo.

\title{
SUMMARY: VARIATIONS OF DISSOLVED ORGANIC CARBON AND SOIL PHYSICAL PROPERTIES UNDER DIFFERENT LAND USES IN CENTRAL AMAZONIA
}

\begin{abstract}
The purpose of this study was to assess the relationship between dissolved organic carbon (DOC) and physics properties of soil under different land use systems in central Amazonia. The DOC concentrations of primary forest soil were measured in three topographical positions, and in areas of pasture, secondary forest and an agroforestry system (AFs) down to a depth of $2 \mathrm{~m}$. The soil solution was collected and analyzed for the organic $C$ phase during a hydrological cycle. The average dissolved organic carbon (DOC) concentrations followed the sequence: AFs $>$ secondary forest $>$ forest (plateau) > pasture (dry and rainy-dry period), except in the rainy and dry-rainy period, DOC concentration of the pasture soil was equal to or higher than that of other environments in certain layers. Results indicated the capacity of the AFs and secondary forest to recover organic C and/or make it available in soil solution. These are the recommended land use forms to maintain carbon in the terrestrial environment. The DOC was influenced by time, space and depth and decreased with depth. The soil structure, represented by soil aggregation, total organic carbon (TOC) and porosity, exerted great influence on the DOC concentrations in the soil layers and, together with the more labile soil organic matter, determined carbon maintenance in the soil solution. The DOC concentrations measured under vegetation covers and land uses indicate the importance of adequate soil managements to maintain carbon in the soil ecosystem.
\end{abstract}

Index terms: soil solution, soil carbon dynamics, vegetation cover, soil physics.

\section{INTRODUÇÃO}

A matéria orgânica dissolvida (MOD) representa importante elemento em muitos processos biogeoquímicos do solo e da água (Ussiri \& Johnson, 2004). Ela tem sua origem a partir da lixiviação da liteira (resultante da matéria orgânica particulada) e da atividade da biomassa microbiana do solo (Matlou \& Haynes, 2006). Inúmeros fatores bióticos e abióticos controlam a dinâmica temporal e espacial da MOD (Murphy et al., 2000). Alguns fatores são determinantes para as concentrações do C orgânico dissolvido (COD), como o substrato (liteira e MOS), a composição da comunidade microbiana (Moller et al., 1999), a temperatura, os fluxos de água (Brooks et al., 1999) e as espécies de árvores (Smolander \& Kitunen, 2002), que produzem as variantes químicas da liteira. Por outro lado, as práticas de manejo do solo afetam a MOD (Chantigny, 2003), alterando o estado de equilíbrio do COD.

Nos últimos anos, as pesquisas sobre a dinâmica e o estoque de $\mathrm{C}$ na solução do solo têm recebido atenção especial (Neu, 2005; Matlou \& Haynes, 2006; Eimers et al., 2008). Um número significativo de pesquisas tem sido realizado, principalmente para caracterizar a variabilidade espacial e temporal do COD e dos seus fluxos (Aitkenhead-Peterson et al., 2003) e para quantificar as características químicas e pedogenéticas do solo (Jansen et al., 2003) e a disponibilidade do COD para a microflora do solo (Kalbitz et al., 2003). A grande preocupação está relacionada à contribuição potencial do COD para o aumento das concentrações de $\mathrm{CO}_{2}$ atmosférico, pelo aumento dos fluxos de COD nos igarapés, resultante da exportação natural para a rede de drenagem nos baixios florestais. Esses aumentos têm sido acelerados pelas mudanças do uso da terra, especialmente pela conversão de floresta em pastagens (Ross et al., 1999).

O objetivo deste trabalho foi verificar a relação entre $\mathrm{C}$ orgânico dissolvido (COD) e atributos físicos do solo sob áreas de floresta primária, pastagem, sucessão secundária e sistema agroflorestal na Amazônia central. 


\section{MATERIAL E MÉTODOS}

\section{Localização das coberturas vegetais e sistemas de uso da terra (SUT)}

O estudo foi realizado em três sistemas de uso da terra, distantes $20 \mathrm{~km}$, na Amazônia central: (i) floresta primária, numa catena em diferentes posições topográficas (platô, encosta e baixio), na Reserva Experimental do Cuieiras, no km 34 da estrada vicinal ZF-2, $60 \mathrm{~km}$ ao norte de Manaus, AM (02 $36^{\prime} 32,1^{\prime \prime} \mathrm{S}$ e $\left.60^{\circ} 12^{\prime} 32,4^{\prime \prime} \mathrm{W}\right)$; (ii) pastagem manejada, localizada na Reserva Experimental do Projeto Dinâmica Biológica de Fragmentos Florestais (PDBFF), no km 23 da estrada vicinal ZF-3 ( $02^{\circ} 25^{\prime}$,, $7^{\prime}$ ' S e $\left.59^{\circ} 52^{\prime} 51^{\prime \prime} \mathrm{W}\right)$; e (iii) sistema agroflorestal (SAF) e sucessão secundária, localizados na Estação Experimental da Embrapa Amazônia Ocidental, ao norte de Manaus, no km 54 da BR-174 (2० $32^{\prime}$ S e $60^{\circ} 02^{\prime}$ W). Os solos foram classificados in situ como: Latossolo Amarelo distrófico textura argilosa a muito argilosa (floresta-platô e pastagem), Latossolo VermelhoAmarelo distrófico textura muito argilosa (sucessão secundária e SAF), Argissolo Vermelho-Amarelo distrófico textura argilosa (floresta-encosta) e Espodossolo Cárbico Hidromórfico típico (florestabaixio).

\section{Caracterização dos sistemas de uso da terra (SUT)}

A área de floresta foi caracterizada como Ombrófila Densa, diferenciada em três posições topográficas: floresta de platô com solos de textura muito argilosa (Latossolo Amarelo distrófico), com dossel de 35-40 m; floresta de encosta, em relevo ondulado sobre solos de textura média e argilosa (Argissolo Vermelho-Amarelo distrófico), com dossel de 20-35 m; e floresta de baixio em relevo plano e solos arenosos (Espodossolo Cárbico Hidromórfico típico), com dossel até $30 \mathrm{~m}$.

Pastagem: área manejada em sistema de pastejo rotacional, semiconfinado, em relevo ondulado suave, em solos do tipo Latossolo Vermelho-Amarelo distrófico, com textura muito argilosa, cobertura de gramínea Brachiaria humidicola (quicuio-daamazônia), sem prática de queima no decorrer dos últimos cinco anos. Sistema agroflorestal (SAF): área constituída por sistema agrossilvicultural de 16 anos, dominado por cupuaçuzeiro (Teobroma grandiflorum), pupunheira (Bactris gassipaes), açaizeiro (Euterpe oleraceae) e andiroba (Carapa guianensis), sobre Latossolo Amarelo distrófico, textura muito argilosa. Sucessão secundária: vegetação originada a partir da regeneração natural de pastagem degradada, com 16 anos, dominada por espécies do gênero Vismia, principalmente $V$. japrurensis, $V$. caynensis e $V$. guianensis, por Laetia procera, Belucia glossularioides e B. dichotoma.

\section{Instalação de extratores}

$\mathrm{Na}$ área de floresta primária, instalaram-se os extratores em três posições topográficas distintas: platô, encosta e baixio, nas profundidades de 10, 20, 30, 40, 100 e $200 \mathrm{~cm}$ (platô e encosta), num total de 24 extratores, sendo 12 em cada posição topográfica. No baixio, devido à proximidade do lençol freático, os extratores foram instalados somente até $60 \mathrm{~cm}$ de profundidade e dispostos nas profundidades de $10,20,30,40$ e $60 \mathrm{~cm}$, perfazendo um total de 10 extratores nessa posição topográfica. Nas áreas de pastagem, SAF e sucessão secundária foram instaladas as cápsulas extratoras nas profundidades de 10, 40, 100 e $200 \mathrm{~cm}$, com o intuito de visualizar os contrastes mais intensos das áreas entre si e com a floresta de platô, já que as classes de solos são idênticas. Após a instalação, o sistema foi deixado em equilíbrio por um período de três meses, desprezando-se a primeira coleta.

\section{Coleta da solução do solo}

A solução do solo foi amostrada ao longo de um ciclo hidrológico, considerando-se o período seco (setembro e outubro), período chuvoso (janeiro a maio), transição seco-chuvoso (novembro e dezembro) e transição chuvoso-seco (junho, julho e agosto). As coletas foram realizadas a cada 15 dias, porém não foi possível coletar a solução do solo em todas as profundidades estudadas, devido à escassez dessa solução em determinados dias. As coletas foram realizadas com auxílio de uma bomba de vácuo para promover uma diferença de pressão, aplicando-se uma tensão de $60 \mathrm{kPa}$ no sistema cápsula-solo-cápsula. No campo, as amostras foram armazenadas em recipientes apropriados, previamente descontaminados e conservados bem fechados e sob refrigeração até sua chegada ao Laboratório de Águas do INPA/CPRHC - Coordenação de Pesquisas em Recursos Hídricos e Clima. No ato da coleta da solução, cada amostra era filtrada, utilizando-se filtros com membrana de fibra de vidro com porosidade de $0,7 \mathrm{~mm}$. A solução destinada à análise de COD era armazenada em frascos Nalgene de $60 \mathrm{~mL}$ pré-calcinados e preservada utilizando $\mathrm{HgCl}_{2}$ (cloreto de mercúrio) com concentração de $300 \mathrm{mmol} \mathrm{L}^{-1} \mathrm{de} \mathrm{Hg}$. O sistema coletor era constituído de cápsula porosa, tubo PVC e capilar PU, sendo esse sistema interligado a um frasco erlenmeyer de $250 \mathrm{~mL}$, devidamente fechado com rolha de silicone, por meio de um pequeno furo para a inserção do capilar PU, sendo possível o armazenamento da solução do solo dentro do erlenmeyer. A coleta da solução do solo foi feita com auxílio de seringa, devidamente limpa a cada troca de profundidade. Depois de cada análise, os frascos eram lavados com água destilada, colocados em estufa para evaporação prévia da água e conduzidos à mufla para serem calcinados em temperatura de $500{ }^{\circ} \mathrm{C}$ durante cinco horas. 


\section{Análises do solo}

A determinação das concentrações do $\mathrm{C}$ orgânico dissolvido (COD) foi feita em analisador de $\mathrm{C}$ (Shimadzu, modelo TOC 5000A), no INPA/CPRHC.

A análise de $\mathrm{C}$ total foi realizada em analisador Fisons Instruments NA 1500 NC, utilizandose aproximadamente $25-30 \mathrm{mg}$ de material, previamente macerado em almofariz e passado em peneira de malha de $212 \mathrm{~mm}$, visando à redução de erros associados ao tamanho de grãos.

A análise granulométrica foi realizada pelo método da pipeta (Embrapa, 1997).

A densidade do solo foi determinada em amostras indeformadas coletadas com amostrador tipo Uhland, com anéis volumétricos de $0,05 \mathrm{~m}$ de altura e 0,05 $\mathrm{m}$ de diâmetro ao longo das camadas selecionadas em quintuplicata.

A determinação da distribuição de poros por tamanho foi obtida a partir das amostras utilizadas para obtenção da densidade do solo, com separação entre macro e microporos por drenagem em tensão de $10 \mathrm{kPa}$. A porosidade total foi calculada pela relação entre densidade do solo e densidade de partícula.

A análise de estabilidade de agregados em água foi realizada conforme Embrapa (1997), a partir de amostras coletadas a $10 \mathrm{~cm}$ de profundidade. A estabilidade de agregados foi expressa pelo diâmetro médio geométrico (DMG).

\section{Análise estatística}

Os dados de COD, COT e atributos físicos do solo foram submetidos à análise de variância, e as médias, comparadas pelo teste de Tukey a $5 \%$. Análises de regressões e correlações de Pearson foram realizadas para relacionar o COD com os atributos físicos do solo.

\section{RESULTADOS}

\section{COD em diferentes posições topográficas sob floresta primária}

Identificaram-se três regiões distintas para a distribuição do COD em profundidade ao longo das posições topográficas: 0-30 $\mathrm{cm}$ (superficial), 40-100 cm (intermediária) e $200 \mathrm{~cm}$ (subsuperficial). $\mathrm{Na}$ camada superficial houve maior variação dos teores de COD, enquanto nas camadas intermediária e subsuperficial houve redução gradativa e maior homogeneidade nos teores de COD. No baixio (Figura 1c), a concentração do COD é maior do que no platô (Figura 1a) e encosta (Figura 1b), estabelecendo-se nas camadas superficiais $(0-30 \mathrm{~cm})$ concentrações entre 48,4 e 49,0 $\mathrm{mg} \mathrm{L}^{-1} \mathrm{e}$, na camada subsuperficial $(60 \mathrm{~cm})$, concentrações até $49,0 \mathrm{mg} \mathrm{L}^{-1}$. $\mathrm{Na}$ camada superficial $(0-30 \mathrm{~cm})$ do platô e na encosta, as concentrações de COD foram menores. No platô (Latossolo), variou de 1,3 a 12,1 $\mathrm{mg} \mathrm{L}^{-1}$ (Figura 1a), e na encosta (Argissolo), de 2,2 a $13,8 \mathrm{mg} \mathrm{L}^{-1}$ (Figura 1b). A camada intermediária (40-100 cm) apresentou teores de COD entre $4 \mathrm{e}$ $11 \mathrm{mg} \mathrm{L}^{-1}$ (Platô - Latossolo) (Figura 1a) e de $2 \mathrm{e}$ $10 \mathrm{mg} \mathrm{L}^{-1}$ (Encosta - Argissolo) (Figura 1b), com valores reduzidos em relação à superfície. A camada subsuperficial $(200 \mathrm{~cm})$ apresentou valores mais baixos, na ordem de 1,8 a 6,0 mg L-1 (Platô - Latossolo) e de 1,3 a 10,0 $\mathrm{mg} \mathrm{L}^{-1}$ (Encosta - Argissolo). Na escala temporal, as concentrações de COD aumentaram com o aumento das chuvas (Figura 1a,b,c). As maiores concentrações de COD foram encontradas a $10 \mathrm{~cm}$ nos solos de textura média (Encosta/ Argissolo) a muito argilosa (Platô/Latossolo), sendo 13,8 e $12,1 \mathrm{mg} \mathrm{L}^{-1}$, respectivamente. A $200 \mathrm{~cm}$ de profundidade o COD reduz-se drasticamente, principalmente no período seco, variando de 3,0 a $4,0 \mathrm{mg} \mathrm{L}^{-1}$. (Figura 1a,b). As camadas mais profundas da Encosta-Argissolo (Figura 1b) mostraram COD mais elevado do que no Platô - Latossolo (Figura 1a).

\section{COD sob floresta (platô), pastagem, sistema agrofloresal (SAF) e sucessão secundária}

As concentrações de COD seguiram a ordem SAF $>$ sucessão secundária $>$ floresta (topo) > pastagem. As concentrações de COD nas camadas de 10, 20, 40 e $200 \mathrm{~cm}$ no Latossolo Amarelo sob pastagem oscilaram entre 1,5 e 14,6 $\mathrm{mg} \mathrm{L}^{-1}$; no SAF, de 4,0 a $24,8 \mathrm{mg} \mathrm{L}^{-1}$; na sucessão secundária as concentrações de COD foram mais altas $\left(3,0\right.$ a $\left.23,0 \mathrm{mg} \mathrm{L}^{-1}\right)$ do que as encontradas na pastagem (Figura 2). Os teores de COD em pastagem $\left(1,5\right.$ a $\left.14,6 \mathrm{mg} \mathrm{L}^{-1}\right)$, sistema agroflorestal (3,8 a $\left.24,8 \mathrm{mg} \mathrm{L}^{-1}\right)$ e sucessão secundária (3,0 a 23,0 $\left.\mathrm{mg} \mathrm{L}^{-1}\right)$ diminuíram em profundidade (Figura 2). Os maiores teores de COD foram obtidos nas camadas superficiais, em todos os ambientes estudados, diferenciando-se significativamente das outras profundidades (Figura 3).

\section{COD e atributos físicos do solo}

As correlações entre COD e atributos do solo sofreram variações devido à época amostrada, profundidade e uso da terra. No SAF e sucessão secundária, o COD demonstrou sofrer influência direta e positiva do DMG e COT em todas as épocas e negativamente com o teor de argila, exceto no período seco-chuvoso. Na pastagem, o COD foi influenciado positivamente pelo DMG, pela porosidade total e pelo COT, em todas as épocas, exceto pelo COT obtido no período seco-chuvoso; 

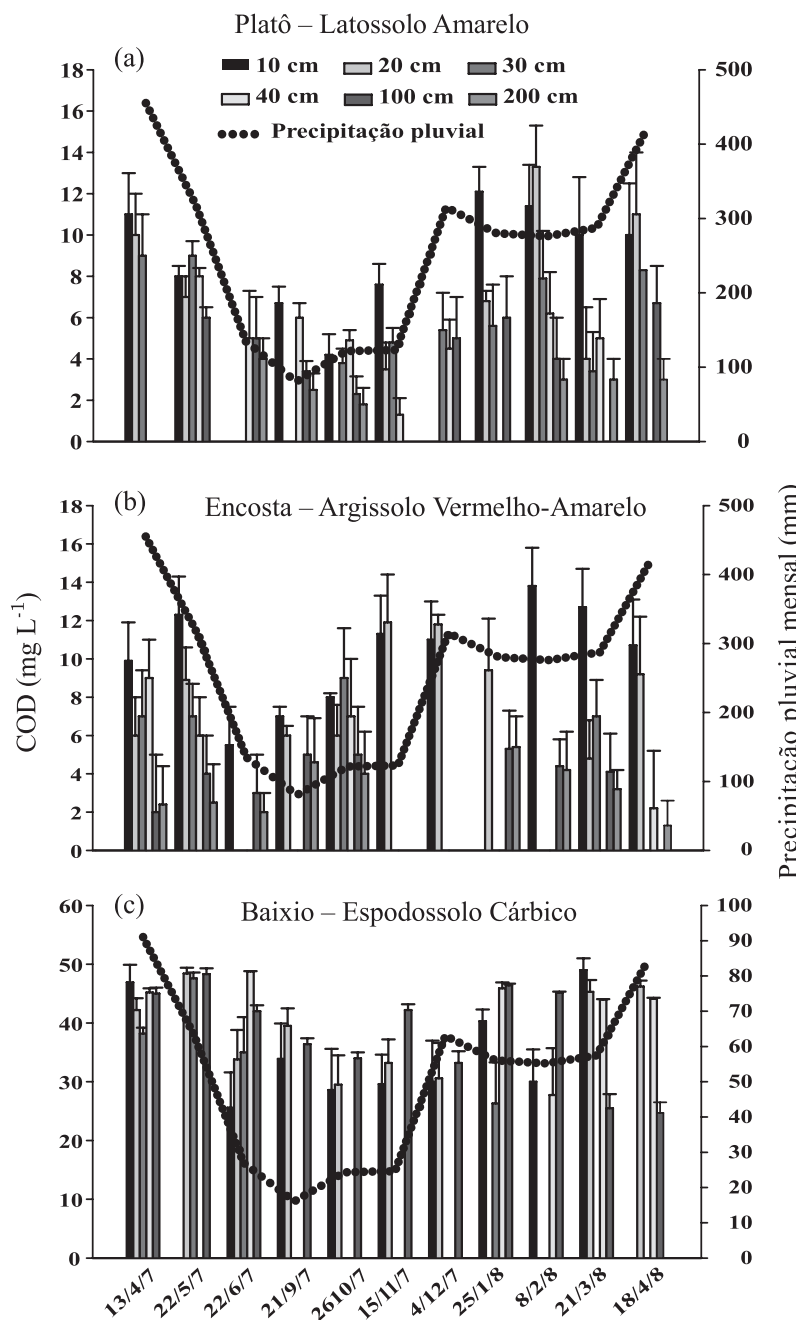

Mês/ano

Figura 1. Variação do COD em profundidade associada à precipitação pluvial sob floresta primária, em diferentes posições topográficas: platô (a), encosta (b) e baixio (c). As barras nos gráficos representam as coletas de COD realizadas, por profundidade, com seus respectivos desvios-padrão, as datas de coleta e as profundidades; a linha pontilhada mostra a precipitação pluvial de cada período.

constatou-se correlação negativa com o teor de argila no período chuvoso e seco-chuvoso. Na floresta primária (platô e encosta), o COD correlacionouse direta e positivamente com o COT, exceto nos períodos seco-chuvoso e chuvoso, e com o COT obtido na floresta (baixio) em todas as épocas amostradas. O COD só demonstrou correlação direta com as variações do DMG na floresta (platô), exceto no período chuvoso (Quadro 1).

Quanto às correlações negativas, verificou-se essa tendência entre o COD e teor de argila e densidade do solo na floresta (platô); entre COD e teor de argila e microporosidade na floresta (encosta), exceto no período seco-chuvoso; e entre COD e densidade do solo, exceto no período chuvoso-seco (Quadro 1).

Esperava-se a obtenção dessa heterogeneidade nas correlações, pois as variações no comportamento do COD são o resultado da sua interação dinâmica com sazonalidade, física do solo (Quadro 2), camadas, saturação e insaturação do solo, sendo pronunciada em solos tropicais em decorrência dos diferentes manejos a que são submetidos.

\section{DISCUSSÃO}

Variações do carbono orgânico dissolvido (COD) em diferentes posições topográficas sob floresta primária

Diferenças nas concentrações de COD nos perfis sob floresta evidenciaram diferentes zonas de
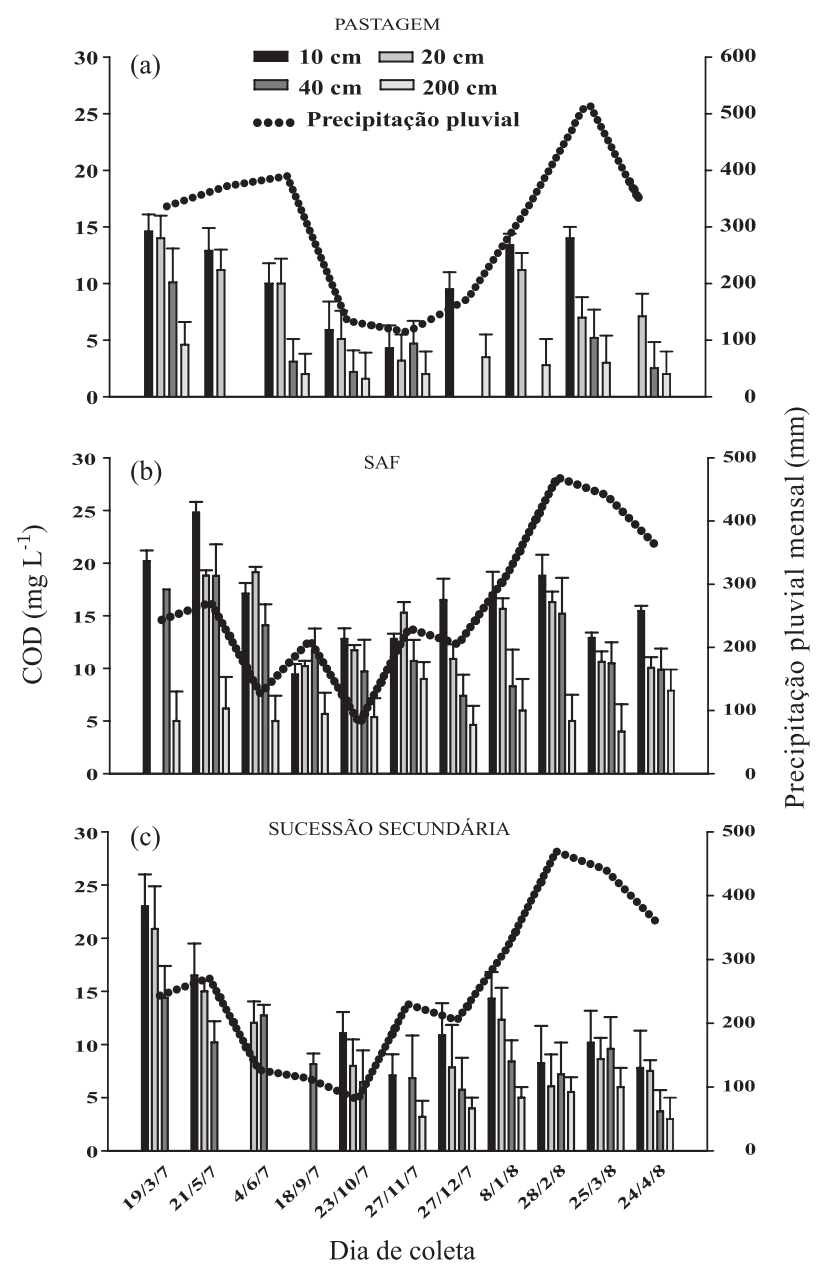

Figura 2. Variação do COD em diferentes épocas e profundidades do solo em pastagem, SAF e sucessão secundária. As barras representam as concentrações de COD na solução do solo com os respectivos desvios-padrão; a linha pontilhada mostra a precipitação pluvial em cada período de coleta. 

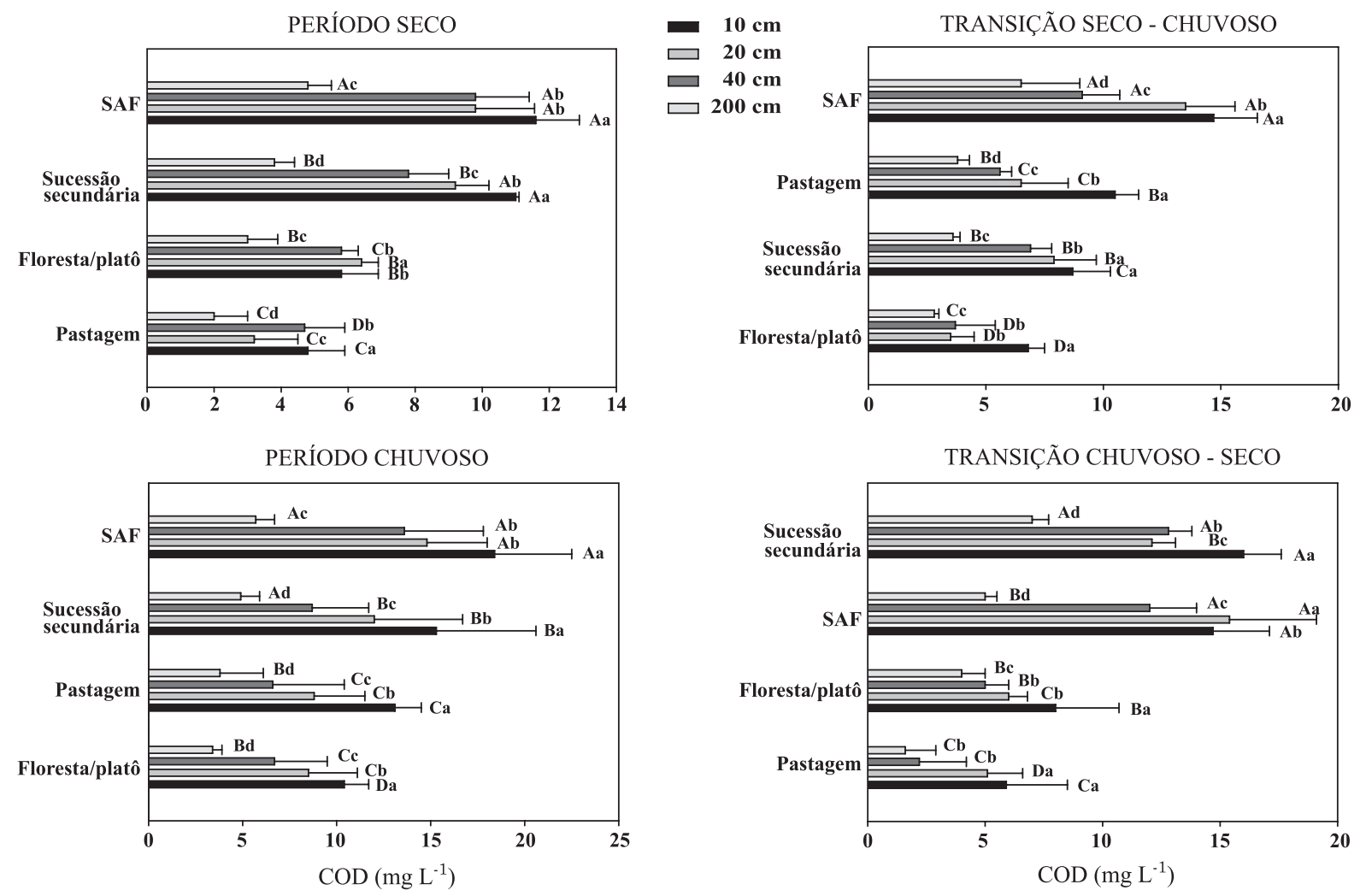

Figura 3. Concentrações de COD na solução do solo em diferentes períodos do ano (seco, chuvoso e transições). A letra maiúscula compara as profundidades semelhantes entre os ambientes, e a minúscula, as profundidades diferentes entre si dentro do mesmo ambiente. (Tukey, p $<0,05)$.

transporte deste no solo (superficial, intermediária e subsuperficial). Entretanto, as concentrações de COD não sofrem variações elevadas entre camadas próximas, demonstrando que o seu transporte é lento, em decorrência da alta retenção de água nos solos argilosos da Amazônia. As altas concentrações de COD que atingem o solo em área de floresta, originadas do dossel e da MOS, diminuem rapidamente na solução do solo com a percolação da água (Qualls et al., 2002). As concentrações do COD geralmente diminuem com a profundidade, devido à retenção que ocorre na superfície do solo, e o mesmo ocorre com os fluxos de COD; a sorção do COD pela matriz do solo provavelmente conduz a grandes estoques de $\mathrm{C}$, especialmente na zona de ação das raízes. A partir de $40 \mathrm{~cm}$ de profundidade, a maior proteção do $\mathrm{C}$ e a retenção da água do solo em solos argilosos fazem com que o COD se movimente lentamente (Johnson et al., 2000). No período chuvoso, o COD apresenta concentrações mais elevadas em profundidade do que no período seco; nas camadas superiores, não sofre grandes alterações entre os períodos seco e chuvoso. Em pesquisas realizadas na Amazônia central, ao norte de Manaus, sob solos argilosos, Neu (2005) constatou também aumento do COD em profundidade em áreas de floresta primária, reflorestamento e floresta secundária, justificando os resultados pela capacidade das raízes em transportar $\mathrm{C}$ no perfil do solo. Da mesma forma, Moller et al. (2005), nas camadas superficiais em plantação de Pinus, campo cultivado e floresta primária, mediram concentrações de COD entre 7,9 e $13,9 \mathrm{mg} \mathrm{L}^{-1}$, próximas às do presente estudo.

A baixa capacidade de adsorção de solos arenosos como o Espodossolo Cárbico (baixio) torna o COD prontamente disponível para a solução do solo. As concentrações máximas do COD em profundidade nessa classe de solo, a 30 e $60 \mathrm{~cm}$ de profundidade (49,0 $\mathrm{mg} \mathrm{L}^{-1}$ ), respectivamente, são explicadas pela ação da liteira, na superfície, e do lençol freático, contribuindo para o enriquecimento de $\mathrm{C}$ oriundo do horizonte espódico, encontrado, neste estudo, a 1,20 m de profundidade. O lençol freático é, então, o responsável pela liberação do $\mathrm{C}$ acumulado via aumento do nível do igarapé no período de cheia (Waterloo et al., 2006). Ainda nesse ambiente, a maior variação da concentração de COD na solução do solo encontrada na camada de $0-30 \mathrm{~cm}$ (49,0 $\left.\mathrm{mg} \mathrm{L}^{-1}\right)$ deve ser decorrente de uma intensa atividade biológica, da decomposição da liteira e do sistema radicular, proporcionando maior eficiência no transporte do COD. As altas concentrações de COD nos igarapés de água escura sob a ação 
Quadro 1. Coeficiente de correlação de Pearson entre as concentrações de COD (mg $\left.\mathrm{L}^{-1}\right)$ e os atributos físicos do solo sob floresta, pastagem, SAF e sucessão secundária, na Amazônia central, nas camadas de 10 a $200 \mathrm{~cm}$, em diferentes períodos do ano

\begin{tabular}{|c|c|c|c|c|}
\hline \multirow{2}{*}{ Atributo do solo } & \multicolumn{4}{|c|}{ Período do ano } \\
\hline & Chuvoso & Seco & Seco - chuvoso & Chuvoso - seco \\
\hline & \multicolumn{4}{|c|}{ Floresta primária - Encosta } \\
\hline Teor de argila $\left(\mathrm{g} \mathrm{kg}^{-1}\right)$ & $-0,67$ & $-0,64$ & $-0,65$ & $-0,93$ \\
\hline Densidade do solo $\left(\mathrm{g} \mathrm{cm}^{-3}\right)$ & $-0,61$ & $-0,61$ & $-0,46$ & $-0,84$ \\
\hline Porosidade total $\left(\mathrm{m}^{-3} \mathrm{~m}^{-3}\right)$ & $\mathrm{ns}$ & 0,34 & 0,40 & $\mathrm{~ns}$ \\
\hline Macroporosidade $\left(\mathrm{m}^{-3} \mathrm{~m}^{-3}\right)$ & $\mathrm{ns}$ & $\mathrm{ns}$ & $-0,69$ & $\mathrm{~ns}$ \\
\hline Microporosidade $\left(\mathrm{m}^{-3} \mathrm{~m}^{-3}\right)$ & $-0,48$ & $-0,50$ & $-0,48$ & $-0,63$ \\
\hline Carbono orgânico total (\%) & 0,70 & 0,50 & 0,41 & 0,60 \\
\hline \multirow[t]{2}{*}{ Diâmetro médio geométrico $(\mathrm{mm})$} & ns & 0,77 & 0,60 & 0,74 \\
\hline & \multicolumn{4}{|c|}{ Floresta primária - Encosta } \\
\hline Teor de argila $\left(\mathrm{g} \mathrm{kg}^{-1}\right)$ & $-0,84$ & $-0,76$ & - & $-0,84$ \\
\hline Densidade do solo $\left(\mathrm{g} \mathrm{cm}^{-3}\right)$ & $-0,33$ & ns & - & ns \\
\hline Porosidade total $\left(\mathrm{m}^{-3} \mathrm{~m}^{-3}\right)$ & 0,44 & 0,48 & - & $-0,53$ \\
\hline Macroporosidade $\left(\mathrm{m}^{-3} \mathrm{~m}^{-3}\right)$ & 0,40 & 0,62 & - & $-0,64$ \\
\hline Microporosidade $\left(\mathrm{m}^{-3} \mathrm{~m}^{-3}\right)$ & $-0,56$ & $-0,51$ & - & $-0,60$ \\
\hline Carbono orgânico total (\%) & 0,46 & 0,50 & 0,60 & 0,75 \\
\hline \multirow[t]{2}{*}{ Diâmetro médio geométrico $(\mathrm{mm})$} & 0,50 & $\mathrm{~ns}$ & $\mathrm{~ns}$ & 0,60 \\
\hline & \multicolumn{4}{|c|}{ Floresta primária - Baixio } \\
\hline Teor de argila $\left(\mathrm{g} \mathrm{kg}^{-1}\right)$ & $\mathrm{ns}$ & $\mathrm{ns}$ & $\mathrm{ns}$ & $\mathrm{ns}$ \\
\hline Densidade do solo $\left(\mathrm{g} \mathrm{cm}^{-3}\right)$ & $-0,81$ & $-0,70$ & $-0,96$ & $-0,48$ \\
\hline Porosidade total $\left(\mathrm{m}^{-3} \mathrm{~m}^{-3}\right)$ & ns & ns & $\mathrm{ns}$ & ns \\
\hline Macroporosidade $\left(\mathrm{m}^{-3} \mathrm{~m}^{-3}\right)$ & 0,86 & $\mathrm{~ns}$ & ns & 0,84 \\
\hline Microporosidade $\left(\mathrm{m}^{-3} \mathrm{~m}^{-3}\right)$ & $\mathrm{ns}$ & ns & $\mathrm{ns}$ & ns \\
\hline Carbono orgânico total (\%) & 0,80 & 0,65 & 0,65 & 0,71 \\
\hline \multirow{2}{*}{ Diâmetro médio geométrico $(\mathrm{mm})$} & - & - & - & - \\
\hline & \multicolumn{4}{|c|}{ Pastagem } \\
\hline Teor de argila $\left(\mathrm{g} \mathrm{kg}^{-1}\right)$ & $-0,50$ & $-0,48$ & $-0,54$ & $-0,42$ \\
\hline Densidade do solo $\left(\mathrm{g} \mathrm{cm}^{-3}\right)$ & $-0,38$ & $-0,45$ & $-0,41$ & $\mathrm{~ns}$ \\
\hline Porosidade total $\left(\mathrm{m}^{-3} \mathrm{~m}^{-3}\right)$ & 0,78 & 0,63 & 0,83 & 0,67 \\
\hline Macroporosidade $\left(\mathrm{m}^{-3} \mathrm{~m}^{-3}\right)$ & 0,61 & 0,69 & 0,62 & 0,71 \\
\hline Microporosidade $\left(\mathrm{m}^{-3} \mathrm{~m}^{-3}\right)$ & 0,77 & 0,67 & 0,81 & 0,65 \\
\hline Carbono orgânico total (\%) & 0,70 & 0,70 & 0,44 & 0,65 \\
\hline \multirow[t]{2}{*}{ Diâmetro médio geométrico $(\mathrm{mm})$} & 0,60 & 0,75 & 0,70 & 0,71 \\
\hline & \multicolumn{4}{|c|}{ Sistema agroflorestal - SAF } \\
\hline Teor de argila $\left(\mathrm{g} \mathrm{kg}^{-1}\right)$ & $-0,62$ & $-0,69$ & 0,83 & $-0,55$ \\
\hline Densidade do solo $\left(\mathrm{g} \mathrm{cm}^{-3}\right)$ & $-0,46$ & $-0,51$ & $-0,50$ & $-0,42$ \\
\hline Porosidade total $\left(\mathrm{m}^{-3} \mathrm{~m}^{-3}\right)$ & 0,22 & 0,20 & 0,28 & 0,10 \\
\hline Macroporosidade $\left(\mathrm{m}^{-3} \mathrm{~m}^{-3}\right)$ & 0,26 & 0,93 & 0,24 & 0,47 \\
\hline Microporosidade $\left(\mathrm{m}^{-3} \mathrm{~m}^{-3}\right)$ & $-0,45$ & $-0,51$ & $-0,51$ & $-0,50$ \\
\hline Carbono orgânico total (\%) & 0,84 & 0,60 & 0,80 & 0,93 \\
\hline \multirow[t]{2}{*}{ Diâmetro médio geométrico $(\mathrm{mm})$} & 0,60 & 0,80 & 0,71 & 0,80 \\
\hline & \multicolumn{4}{|c|}{ Sucessão secundária } \\
\hline Teor de argila $\left(\mathrm{g} \mathrm{kg}^{-1}\right)$ & $-0,54$ & $-0,58$ & $-0,60$ & $-0,62$ \\
\hline Densidade do solo $\left(\mathrm{g} \mathrm{cm}^{-3}\right)$ & $-0,60$ & $-0,67$ & $-0,71$ & $-0,46$ \\
\hline Porosidade total $\left(\mathrm{m}^{-3} \mathrm{~m}^{-3}\right)$ & 0,48 & 0,50 & 0,52 & 0,22 \\
\hline Macroporosidade $\left(\mathrm{m}^{-3} \mathrm{~m}^{-3}\right)$ & 0,70 & 0,80 & 0,85 & 0,26 \\
\hline Microporosidade $\left(\mathrm{m}^{-3} \mathrm{~m}^{-3}\right)$ & $-0,47$ & $-0,50$ & $-0,51$ & $-0,45$ \\
\hline Carbono orgânico total (\%) & 0,81 & 0,70 & 0,70 & 0,80 \\
\hline Diâmetro médio geométrico $(\mathrm{mm})$ & 0,62 & 0,75 & 0,62 & 0,82 \\
\hline
\end{tabular}

Correlação significativa a 5 \%; ns: não significativo; (-) amostra não coletada.

de gradientes topográficos podem também ser atribuídas à drenagem dos Espodossolos, que apresentam menor capacidade de adsorção do COD, uma vez que a mobilidade deste é controlada pela sorção à superfície dos minerais de argila (McClain et al., 1997). Os resultados obtidos concordam com os de Neu (2009), que obteve grande variação do COD ao longo do ano e entre profundidades em área de floresta na bacia do alto Xingu. Entretanto, divergem devido aos teores de COD, talvez em função 
Quadro 2. Atributos físicos e carbono orgânico total e dissolvido do solo sob floresta, pastagem, sucessão secundária e SAF, na Amazônia central

\begin{tabular}{|c|c|c|c|c|c|c|c|c|}
\hline Área & Profundidade & Argila & $\rho$ & $\mathbf{P t}$ & Ma & Mi & DMG & COT \\
\hline & $\mathrm{cm}$ & $\mathrm{g} \mathrm{kg}^{-1}$ & $\mathrm{~g} \mathrm{~cm}^{-3}$ & 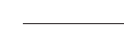 & $-m^{3} m^{-3}$ & - & $\mathrm{mm}$ & $\mathrm{g} \mathrm{kg}^{-1}$ \\
\hline \multirow{6}{*}{ Floresta Platô } & 10 & $695 \mathrm{c}$ & $1,07 \mathrm{c}$ & $0,55 \mathrm{a}$ & $0,12 \mathrm{a}$ & $0,42 \mathrm{c}$ & $3,2 \mathrm{a}$ & $30 \mathrm{a}$ \\
\hline & 20 & $794 \mathrm{~b}$ & $1,11 \mathrm{c}$ & $0,48 \mathrm{~d}$ & $0,02 \mathrm{c}$ & $0,46 \mathrm{a}$ & - & $18 \mathrm{~b}$ \\
\hline & 30 & 802 a & $1,10 \mathrm{c}$ & $0,50 \mathrm{bcd}$ & $0,03 \mathrm{c}$ & $0,47 \mathrm{a}$ & - & $15 \mathrm{c}$ \\
\hline & 40 & $828 \mathrm{a}$ & $1,18 \mathrm{~b}$ & $0,51 \mathrm{bcd}$ & $0,06 \mathrm{~b}$ & $0,44 \mathrm{~b}$ & - & $10 \mathrm{~d}$ \\
\hline & 100 & 859 a & $1,26 \mathrm{a}$ & $0,52 \mathrm{bc}$ & $0,05 \mathrm{~b}$ & $0,47 \mathrm{a}$ & - & $5 \mathrm{e}$ \\
\hline & 200 & $868 \mathrm{a}$ & $1,24 \mathrm{a}$ & $0,53 \mathrm{a}$ & $0,05 \mathrm{~b}$ & $0,48 \mathrm{a}$ & - & $3 \mathrm{f}$ \\
\hline \multirow[t]{6}{*}{ Floresta Encosta } & 10 & $310 \mathrm{c}$ & $1,37 \mathrm{~d}$ & $0,49 \mathrm{bc}$ & $0,21 \mathrm{a}$ & $0,28 \mathrm{bc}$ & $0,7 \mathrm{c}$ & $26 \mathrm{a}$ \\
\hline & 20 & $431 \mathrm{~b}$ & $1,46 \mathrm{~b}$ & $0,46 \mathrm{~d}$ & $0,19 \mathrm{~b}$ & $0,27 \mathrm{c}$ & - & $16 \mathrm{~b}$ \\
\hline & 30 & $431 \mathrm{~b}$ & $1,50 \mathrm{a}$ & $0,45 \mathrm{~d}$ & $0,12 \mathrm{~d}$ & $0,32 \mathrm{a}$ & - & $13 \mathrm{c}$ \\
\hline & 40 & $434 \mathrm{~b}$ & $1,51 \mathrm{a}$ & $0,52 \mathrm{ab}$ & $0,21 \mathrm{a}$ & $0,30 \mathrm{~b}$ & - & $8 \mathrm{~d}$ \\
\hline & 100 & $482 \mathrm{a}$ & $1,42 \mathrm{c}$ & $0,54 \mathrm{a}$ & $0,22 \mathrm{a}$ & $0,32 \mathrm{a}$ & - & 3 e \\
\hline & 200 & $467 \mathrm{a}$ & $1,51 \mathrm{a}$ & $0,51 \mathrm{bc}$ & $0,17 \mathrm{c}$ & $0,33 \mathrm{a}$ & - & $2 \mathrm{e}$ \\
\hline \multirow[t]{5}{*}{ Floresta Baixio } & 10 & $120 \mathrm{~b}$ & $1,54 \mathrm{~d}$ & $0,49 \mathrm{a}$ & $0,39 \mathrm{a}$ & $0,08 \mathrm{a}$ & - & $45 \mathrm{a}$ \\
\hline & 20 & $170 \mathrm{a}$ & $1,57 \mathrm{bc}$ & $0,46 \mathrm{~b}$ & $0,38 \mathrm{a}$ & $0,08 \mathrm{a}$ & - & $21 \mathrm{~b}$ \\
\hline & 30 & $120 \mathrm{~b}$ & $1,60 \mathrm{~b}$ & $0,39 \mathrm{c}$ & $0,36 \mathrm{~b}$ & $0,02 \mathrm{bc}$ & - & $11 \mathrm{c}$ \\
\hline & 40 & $100 \mathrm{c}$ & $1,68 \mathrm{a}$ & $0,38 \mathrm{c}$ & $0,35 \mathrm{bc}$ & $0,01 \mathrm{c}$ & - & $8 \mathrm{~d}$ \\
\hline & 60 & $100 \mathrm{c}$ & $1,55 \mathrm{c}$ & $0,36 \mathrm{c}$ & $0,33 \mathrm{c}$ & $0,03 \mathrm{~b}$ & - & $6 \mathrm{~d}$ \\
\hline \multirow[t]{4}{*}{ Pastagem } & 10 & $800 \mathrm{~d}$ & $1,10 \mathrm{~d}$ & $0,56 \mathrm{a}$ & $0,05 \mathrm{a}$ & $0,50 \mathrm{a}$ & $2,1 \mathrm{~b}$ & $37 \mathrm{a}$ \\
\hline & 20 & $895 \mathrm{c}$ & $1,18 \mathrm{~b}$ & $0,43 \mathrm{~b}$ & $0,05 \mathrm{a}$ & $0,38 \mathrm{~b}$ & - & $19 \mathrm{~b}$ \\
\hline & 40 & $906 \mathrm{~b}$ & $1,15 \mathrm{c}$ & $0,40 \mathrm{~b}$ & $0,03 \mathrm{~b}$ & $0,37 \mathrm{~b}$ & - & $10 \mathrm{c}$ \\
\hline & 200 & $921 \mathrm{a}$ & $1,21 \mathrm{a}$ & $0,37 \mathrm{c}$ & $0,04 \mathrm{ab}$ & $0,33 \mathrm{c}$ & - & $4 \mathrm{~d}$ \\
\hline \multirow[t]{4}{*}{ Sucessão Secundária } & 10 & $775 \mathrm{~d}$ & $1,09 \mathrm{c}$ & $0,59 \mathrm{a}$ & $0,03 \mathrm{a}$ & $0,55 \mathrm{a}$ & $3,7 \mathrm{a}$ & $43 \mathrm{a}$ \\
\hline & 20 & $827 \mathrm{c}$ & $1,16 \mathrm{~b}$ & $0,57 \mathrm{a}$ & $0,03 \mathrm{a}$ & $0,54 \mathrm{a}$ & - & $18 \mathrm{~b}$ \\
\hline & 40 & $855 \mathrm{~b}$ & $1,16 \mathrm{~b}$ & $0,53 \mathrm{~b}$ & $0,03 \mathrm{a}$ & $0,50 \mathrm{~b}$ & - & $11 \mathrm{c}$ \\
\hline & 200 & $900 \mathrm{a}$ & $1,31 \mathrm{a}$ & $0,53 \mathrm{~b}$ & $0,02 \mathrm{a}$ & $0,50 \mathrm{~b}$ & - & $3 \mathrm{~d}$ \\
\hline \multirow[t]{4}{*}{ SAF } & 10 & $741 \mathrm{c}$ & $1,18 \mathrm{c}$ & $0,57 \mathrm{a}$ & $0,08 \mathrm{a}$ & $0,48 \mathrm{~b}$ & $3,8 \mathrm{a}$ & $41 \mathrm{a}$ \\
\hline & 20 & $865 \mathrm{~b}$ & $1,26 \mathrm{~b}$ & $0,52 \mathrm{c}$ & $0,05 \mathrm{~b}$ & $0,46 \mathrm{c}$ & - & $17 \mathrm{~b}$ \\
\hline & 40 & $878 \mathrm{~b}$ & $1,27 \mathrm{~b}$ & $0,52 \mathrm{c}$ & $0,02 \mathrm{c}$ & $0,49 a b$ & - & $11 \mathrm{c}$ \\
\hline & 200 & $910 \mathrm{a}$ & $1,32 \mathrm{a}$ & $0,54 \mathrm{~b}$ & $0,02 \mathrm{c}$ & $0,52 \mathrm{a}$ & - & $3 \mathrm{~d}$ \\
\hline
\end{tabular}

Os valores são médias de cinco amostras $(n=5)$. SAF: sistema agroflorestal; $\rho$ : densidade do solo; Pt: porosidade total; Ma: macroporosidade; Mi: microporosidade; DMG: diâmetro médio geométrico das partículas do solo, determinado e comparado entre os SUTs somente a $10 \mathrm{~cm}$ de profundidade; COT: C orgânico total; COD: carbono orgânico dissolvido. (-) dados não coletados ou não determinados. Médias seguidas de letras diferentes nas colunas indicam diferença significativa entre as profundidades pelo teste de Tukey a de $5 \%$.

da classe de solo e vegetação. Pequenas variações na textura, estrutura do solo, COT e qualidade da liteira podem interferir no deslocamento do C, implicando diferenças nas variações de COD ao longo do perfil de solo. McClain et al. (1997) observaram variabilidade em horizontes minerais em solos arenosos com concentrações entre 35,9 e $38 \mathrm{mg} \mathrm{L}^{-1}$. Outras pesquisas descrevem altas variações nas concentrações de COD em lençóis freáticos (Waterloo et al., 2006), concordando com as variações de COD observadas nesta pesquisa.

O C orgânico particulado também contribui significativamente para as flutuações do COD nos solos, por meio do material detrítico (necromassa), que é, na maioria dos casos, rapidamente mineralizado, aumentando a concentração do COD. A lixiviação de material orgânico no horizonte $\mathrm{O}$ resulta em solução do solo com alto COD e baixas concentrações de metais (Ussiri \& Johnson, 2004). A similaridade das concentrações de COD entre platô e encosta sob floresta demonstra a importância da cobertura vegetal na manutenção do $\mathrm{C}$ nos ambientes de floresta. As camadas mais profundas na encosta mostraram COD mais elevado do que no platô, apesar de os fluxos de COD na solução do solo tenderem a um decréscimo em profundidade, sugerindo que esse C possa estar sendo exportado, via solução do solo, do platô para o baixio, onde foram encontradas as maiores concentrações (49,0 $\mathrm{mg} \mathrm{L}^{-1}$ ); daí, o COD poderia chegar mais facilmente até a drenagem. As concentrações de $\mathrm{C}$ e $\mathrm{N}$ nas vertentes que drenam água das áreas de captação florestais levam significativas quantidades de matéria orgânica dissolvida para o lençol a grande profundidade nas florestas (Buffam et al., 2001).

Da mesma forma, a queda e deposição de liteira promovem o acúmulo de $\mathrm{C}$ e nutrientes para serem absorvidos pelas plantas e disponibilizados ao longo do perfil do solo através da lixiviação, sendo a liteira um fator importante no controle do estoque 
e da qualidade da matéria orgânica no solo (MOS) (Shepherd et al., 2001), que variam com a textura do solo e com a capacidade de adsorção da MO pelo solo. Outro fator de relevância em solos tropicais para o transporte - ação de misturar e deslocar o material orgânico e mineral do solo da superfície e do subsolo - é a bioturbação (Muggler \& Buurman, 2000; Silva \& Mendonça, 2007). A bioturbação promovida por minhocas, minhocuçus, formigas e térmitas merece destaque na paisagem brasileira (Resende et al., 2007), tendo sido identificada nos solos estudados.

\section{COD sob diferentes sistemas de cobertura e uso da terra na Amazônia}

A cobertura vegetal e a forma de uso do solo foram os fatores mais importantes na dinâmica do COD, sendo mais evidenciado nos ambientes de SAF e sucessão secundária. Nesses ambientes, o manejo do solo influencia diretamente as concentrações de COD na superfície do solo. Apesar de grande parte do COD ser considerado derivado da matéria orgânica mineralizada com taxa lenta de incorporação das fontes recentemente depositadas (Karltun et al., 2005), o SAF e a sucessão secundária (com 15-20 anos de implantação) superaram a produção de COD da floresta primária/platô, nas camadas de $10,20,40$ e $200 \mathrm{~cm}$, nos períodos seco, chuvoso e chuvoso-seco, exceto no período seco-chuvoso, em que a pastagem superou a sucessão secundária a 10 e $200 \mathrm{~cm}$ de profundidade. No SAF, a liteira abundante, diversificada com adições de adubos verdes de leguminosas (boa qualidade química), proporciona condições para a atividade biológica e a formação de MO (Tapia-Coral et al., 2005; Luizão et al., 2006). Na sucessão secundária, a liteira, predominantemente de difícil decomposição (Mesquita et al., 1998), favorece a maior humificação e acúmulo de $\mathrm{C}$ no solo. Da mesma forma, a contínua entrada da liteira na superfície do solo e os diferentes sistemas radiculares em profundidade podem ter contribuído para que o COD nesses dois ambientes superasse o encontrado no solo da floresta primária.

Luizão et al. (2004) descrevem a importância da contribuição dos altos teores de $\mathrm{C}$ da liteira para os solos de terra firme na Amazônia. A diversidade da qualidade da liteira produzida, a produção de resíduos orgânicos em diferentes épocas do ano e a macroporosidade do solo, criada e mantida pela ação do sistema radicular, no solo sob as espécies cultivadas no SAF e das nativas da sucessão secundária, são fatores que também contribuem para as maiores concentrações de COD nesses ambientes. A rotação de coberturas vegetais tem muitos efeitos nas concentrações de $\mathrm{C}$ e $\mathrm{N}$ do solo e no COD, devido às diferenças na qualidade $\mathrm{e}$ quantidade dos resíduos produzidos pela cobertura vegetal. Na pastagem, a alta taxa de reposição do $\mathrm{C}$ na superfície, na superfície $(0-10 \mathrm{~cm})$, pela atividade do sistema radicular das gramíneas, mantém o COD elevado e superior ao da floresta, a $10 \mathrm{~cm}$ de profundidade, no período chuvoso. Por outro lado, no período seco, as raízes das plantas na floresta transportam COD com maior eficácia, mantendo maiores concentrações de COD do que a pastagem. A $200 \mathrm{~cm}$ de profundidade, o COD na floresta reduz, principalmente no período chuvoso e seco-chuvoso, mais que na pastagem. Essa diminuição pode ser atribuída à baixa disponibilidade de exsudatos orgânicos nessas camadas da floresta, coincidindo com os estudos realizados por Fiedler \& Kalbitz (2003) e Schwesig et al. (2003). O sistema radicular e a diversidade na qualidade da liteira dos ambientes de SAF e sucessão secundária parecem ser os responsáveis pelos maiores teores de COD no solo desses ambientes, o que, aliado ao tempo de seu estabelecimento, ocasiona a recuperação do solo e a introdução de $\mathrm{C}$ via decomposição dos resíduos orgânicos. Por outro lado, os SUTs estudados podem não ter ainda uma fauna de decompositores tão eficiente como a da floresta e, portanto, apresentar menor mineralização da $\mathrm{MO}$ e, consequentemente, maior humificação e formação de COD.

\section{COD e os atributos do solo}

A correlação direta e positiva do COD da solução do solo com o DMG, o COT e a porosidade em algumas épocas do ano, profundidades e ambientes leva a concluir que a estrutura é importante na movimentação do COD. Nos Latossolos amazônicos com alto teor de argila e microporosidade, a agregação das partículas, o COT e a porosidade podem condicionar o movimento do COD. Em solos tropicais a redução do $\mathrm{C}$ orgânico pode estar relacionada à redução da estabilidade de agregados (Pinheiro et al., 2004), favorecendo a erosão e o empobrecimento do solo em nutrientes. O aumento de macroporos nas camadas do solo reduz o contato de solutos orgânicos e inorgânicos com a matriz do solo, disponibilizando o COD com maior facilidade. A concentração de COD correlacionou-se direta e positivamente com os agregados no período seco e chuvoso/seco na sequência: SAF, sucessão secundária, pastagem, floresta/platô; já o COD foi mais sensível às variações do COT no período chuvoso e menos no período seco na sequência SAF, sucessão secundária, pastagem, floresta/ baixio, floresta/encosta e floresta/platô. No período seco, com a indisponibilidade de água, os agregados tornam-se importantes no transporte de COD no perfil de solo. No período chuvoso, o $\mathrm{C}$ proveniente da lavagem da biomassa e dos resíduos orgânicos promove o aumento do COT; consequentemente, esse C é liberado para a solução, o que não ocorre no período seco, devido à menor disponibilidade de água (Kalbitz et al., 2003; Neu, 2005). 
O COD está distribuído em diferentes poros no solo, sendo a coleta nos macroporos realizada com maior facilidade e intensidade. As maiores concentrações de COD no SAF, sucessão secundária e na floresta (na encosta) sugerem que esses ambientes têm atributos físicos do solo que permitem melhor movimentação do COD, superando os processos de adsorção ao longo do perfil. A maior macroporosidade do baixio $\left(0,39 \mathrm{~m}^{3} \mathrm{~m}^{-3}\right)$ nas camadas de $10,20,30$ e $60 \mathrm{~cm}$ e da encosta $\left(0,22 \mathrm{~m}^{3} \mathrm{~m}^{-3}\right)$ nas camadas e 10 , 20, 40, 100 e $200 \mathrm{~cm}$ promoveu rápida movimentação do COD, já que esses poros, teoricamente, podem armazenar grande quantidade de COD. Os solos fortemente agregados recebem permanentemente grandes volumes de precipitação pluvial, que é liberada por meio dos macroporos, permitindo fluxo maior de matéria orgânica dissolvida em quantidade e composição diferente daquela retida nos microporos por forças capilares ao longo das inclinações da matriz do solo (Kaiser \& Guggenberger, 2005). A maior incidência de poros de drenagem na encosta pode ter influenciado na dinâmica do $\mathrm{C}$, aproximando as concentrações de COD entre platô e encosta. A rápida infiltração através dos macroporos no subsolo em florestas tropicais deve-se ao longo tempo de permanência na matriz do solo pela sorção, às condições químicas de equilíbrio e ao aumento gradativo dos poros de retenção de água em profundidade (Kaiser \& Guggenberger, 2005). Da mesma forma, o aumento no conteúdo de COD tem sido atribuído a distúrbios no solo, ao aumento no fluxo de água, à acumulação do material lábil no solo ou à estimulação da atividade microbiana (Chantigny, 2003). Nos Latossolos Amarelos estudados é possível que nas áreas com maior macroporosidade, em solos de terra firme, altamente porosos $\left(>50 \mathrm{~m}^{3} \mathrm{~m}^{-3}\right)$ e permeáveis, os fluxos nos macroporos sejam responsáveis pela maior parte da entrada da matéria orgânica dissolvida na água subterrânea e na superfície. Em profundidade, o aumento da densidade $\left(>1,2 \mathrm{~g} \mathrm{~cm}^{-3}\right)$ e dos microporos $\left(>45 \mathrm{~m}^{3} \mathrm{~m}^{-3}\right)$ reduz o transporte de COD.

Os horizontes dos solos argilosos apresentaram teor de argila $>700 \mathrm{~g} \mathrm{~kg}^{-1}$, alcançando até $800 \mathrm{~g} \mathrm{~kg}^{-1}$ de argila em profundidade. A variação nos teores de COD obtidas entre os ambientes e a grande variância na concentração de COD entre os SUTs em horizontes minerais podem ser atribuídas em parte aos processos de adsorção/dessorção do solo (McClain et al., 1997). A ação da argila em solos amazônicos com predomínio da caulinita permite que o COD seja armazenado por períodos longos, já que esses solos apresentam alta retenção de água (Marques et al., 2010). As concentrações de COD em profundidade parecem estar relacionadas à capacidade desses solos em reter a solução do solo e disponibilizá-la ao longo do tempo no perfil. O horizonte B controla efetivamente as concentrações de COD (McDowell $\&$ Wood, 1984) quando a solução do solo percola em profundidade, reduzindo o COD (Easthouse et al., 1992). A concentração elevada de COD encontrada nas camadas profundas não é, provavelmente, devido à produção elevada do COD nessas camadas, mas resultado de longos tempos de retenção da água (Hongve, 1999). As concentrações de COD obtidas permitem inferir que os resíduos orgânicos subterrâneos podem ser transportados para horizontes subsuperficiais do solo. A presença das raízes faz com que o COD não seja diretamente adsorvido e imobilizado pelas partículas do solo; isso demonstra que o teor de argila pode não ser o único atributo do solo que controla o movimento e a disponibilidade de COD, salientando-se a estrutura do solo, com grande relevância na movimentação do COD.

\section{CONCLUSÕES}

1. As concentrações médias de $\mathrm{C}$ orgânico dissolvido (COD) na solução do solo seguiram a ordem: SAF ou sucessão secundária > floresta (platô) $>$ pastagem (período seco e chuvoso-seco), exceto no período chuvoso, em que a pastagem se iguala à floresta, exceto a $10 \mathrm{~cm}$ de profundidade; e no período seco-chuvoso, em que a pastagem supera a floresta, em todas as profundidades, ultrapassando a sucessão secundária somente a $10 \mathrm{~cm}$ de profundidade.

2. O SAF e a sucessão secundária demonstraram recuperar e, ou, disponibilizar o C na solução do solo, sendo formas de utilização do solo recomendadas para manter o $\mathrm{C}$ no ambiente terrestre.

3. Identificaram-se três zonas distintas de transporte de COD em profundidade no perfil de solo: superficial $(0-30 \mathrm{~cm})$, intermediária (40$100 \mathrm{~cm})$ e subsuperficial $(200 \mathrm{~cm})$.

4. O COD apresentou variação temporal, espacial e em profundidade, sendo o resultado da interação dinâmica com sazonalidade e atributos do solo, demonstrando heterogeneidade em decorrência do tipo de manejo que é utilizado.

\section{AGRADECIMENTOS}

Aos Projetos LBA, PPG7 e PELD e à EMBRAPA/ CPAA, pelo apoio no desenvolvimento desta pesquisa. Ao CNPq, pela concessão da bolsa de doutorado.

\section{LITERATURA CITADA}

AITKENHEAD-PETERSON, J.A.; MCDOWELL, W.H. \& NEFF, J.C. Sources, production, and regulation of allochthonous dissolved organic matter. In: FINDLAY, S., ed. Dissolved organic matter sources, transport, and transformation in aquatic ecosystems. New York, Academic Press, 2003. 234p. 
BROOKS, P.D.; MCKIGHT, D.M. \& BENCALA, K.E. The relationship between soil heterotrophic activity, soil dissolved organic carbon (DOC) leachate, and catchmentscale DOC export in headwater catchments. Water Res. Res., 35:1895-1902, 1999.

BUFFAM, I.; GALLOWAY, J.N.; BLUM, L.K. \& MCGLATHERY, K.J. A stormflow/baseflow comparison of dissolved organic matter concentrations and bioavailability in an Appalachian stream. Biogeochemistry, 53:269-306, 2001.

CHANTIGNY, M.H. Dissolved and water-extractable organic matter in soils: A review on the influence of land use and management practices. Geoderma, 113:357-380, 2003.

EASTHOUSE, K.B.; MUDER, J.; CHRISTOPHERSEN, N. \& SEIP, H.M. Dissolved organic carbon fractions in soil and stream water during variable hydrological conditions at Birkenes, Southern Norway. Water Res. Res., 28:15851596,1992

EIMERS, M.C.; WATMOUGH, S.A.; BUTTLE, J.M. \& DILLON, P.J. Examination of the potential relationship between droughts, sulphate and dissolved organic carbon at a wetland-draining stream. Global Change Biol., 14:938948,2008

EMPRESA BRASILEIRA DE PESQUISA AGROPECUÁRIA EMBRAPA. Centro Nacional de Pesquisa de Solos. Manual de métodos de análise de solo. 2.ed. Rio de Janeiro, 1997. $212 \mathrm{p}$.

FIEDLER, S. \& KALBITZ, K. Concentrations and properties of dissolved organic matter in forest soils as affected by the redox regime. Soil Sci., 168:793-801, 2003.

HONGVE, D. Production of dissolved organic carbon in forested catchments. J. Hydrol., 224:91-99, 1999.

JANSEN, B.; NIEROP, K.G.J. \& VERSTRATEN, J.M. Mobility of $\mathrm{Fe}(\mathrm{II}), \mathrm{Fe}$ (III) and $\mathrm{Al}$ in acidic forest soils mediated by dissolved organic matter: Influence of solution $\mathrm{pH}$ and metal/organic carbon ratios. Geoderma, 113:323-340, 2003.

JOHNSON, C.E.; DRISCOLL, C.T.; SICCAMA, T.G. \& LIKENS, G.E. Element fluxes and landscape position in a northern hardwood forest watershed ecosystem. Ecosystems, 3:159184,2000

KAISER, K. \& GUGGENBERGER, G. Storm flow flushing in a structured soil changes the composition of dissolved organic matter leached into the subsoil. Geoderma, 127:177-187, 2005.

KALBITZ, K.; SCHMERWITZ, J.; SCHWESIG, D. \& MATZNER, E. Biodegradation of soil-derived dissolved organic matter as related to its properties. Geoderma, 113:273-291, 2003.

KARLTUN, E.; HARRISON, A.F.; ALRIKSOON, A.; BRYANT, C.; GARNETT, M.H. \& OLSSON, M.T. Old organic carbon in soil solution DOC after afforestation - evidence from ${ }^{14} \mathrm{C}$ analysis. Geoderma, 127:188-195, 2005.

LUIZÃO, R.C.C.; LUIZÃO, F.J.; PAIVA, R.Q.; MONTEIRO, T.F.; SOUZA, L.S. \& KRUIJT, B. Variation of carbon and nitrogen cycling processes along a topographic gradient in a central amazonian forest. Global Change Biol., 22:592$600,2004$.
LUIZÃO, F.J.; TAPIA-CORAL, S.; GALLARDO-ORDINOLA, J.; SILVA, G.C.; LUIZÃO, R.C.C.; TRUJILLO-CABRERA, L.; WANDELLI, E. \& FERNANDES, E.C.M. Ciclos biogeoquímicos em agroflorestas na Amazônia. In: GAMARODRIGUES, A.C.; BARROS, N.F.; GAMA-RODRIGUES, E.F. \& FREITAS, M.S.M., orgs. Sistemas agroflorestais: Bases científicas para o desenvolvimento sustentável. Campos dos Goytacazes, Universidade Estadual do Norte Fluminense Darcy Ribeiro, 2006. p.87-100.

MATLOU, M.C. \& HAYNES, R.J. Soluble organic matter and microbial biomass $\mathrm{C}$ and $\mathrm{N}$ in soils under pasture and arable management and the leaching of organic $\mathrm{C}, \mathrm{N}$ and nitrate in a lysimeter study. Appl. Soil Ecol., 34:160-167, 2006.

MARQUES, J.D.; TEIXEIRA, W.T.; REIS, A.M.; JUNIOR, O.C.J.; BATISTA, S.M. \& AFONSO, M.A.C.B. Atributos químicos, físico-hídricos e mineralogia da fração argila em solos do Baixo Amazonas: Serra de Parintins. Acta Amaz., 40:1-12, 2010.

McCLAIN, M.E.; RICHEY, J.E.; BRANDES, J.A. \& PIMENTEL, T.P. Dissolved organic matter and terrestrial lotic linkages in the central Amazon basin of Brazil. Global Biogeochem. Cycles, 11:295-311, 1997.

McDOWELL, W.H. \& WOOD, T. Podzolization: Soil processes control dissolved organic carbon concentrations in stream water. Soil Sci., 137:23-32, 1984.

MESQUITA, R.C.G.; WORKMAN, S. \& NEELY, C. Slow litter decomposition in a Cecropia-dominated secondary forest of central Amazonia. Soil Biol. Biochem., 30:167-175, 1998.

MOLLER, J.; MILLER, M. \& KJOLLER, A. Fungal-bacterial interaction on beech leaves: Influence on decomposition and dissolved organic carbon quality. Soil Biol. Biochem., 31:367-374, 1999

MOLLER, A.; KLAUS, K. \& GUGGENBERGER, G. Dissolved organic carbon and nitrogen in precipitation, throughfall, soil solution and stream water of the tropical highlands in northern Thailand. J. Plant Nutr. Soil Sci., 168:649-659, 2005.

MUGGLER, C.C. \& BUURMAN, P. Erosion, sedimentation and pedogenesis in a polygenetic oxisol sequence in Minas Gerais. Catena, 41:3-17, 2000.

MURPHY, D.V.; MACDONALD, A.J.; STOCKDALE, E.A.; GOULDING, K.W.T.; FORTUNE, S.; GAUNT, J.L.; POULTON, P.R.; WAKEFIELD, J.A.; WEBSTER, C.P. \& WILMER, W.S. Soluble organic nitrogen in agricultural soils. Biol. Fert. Soils, 30:374-387, 2000.

NEU, V. Influência da cobertura vegetal na ciclagem de nutrientes via solução do solo na região de Manaus. Piracicaba, Escola Superior de Agricultura Luiz de Queiroz, 2005. 93p. (Tese de Mestrado)

NEU, V. O ciclo do carbono na Bacia do Alto Xingu: Interações entre ambientes terrestres, aquático e atmosférico. Piracicaba, Escola Superior de Agricultura Luiz de Queiroz, 2009. 114p. (Tese de Doutorado)

PINHEIRO, E.F.M.; PEREIRA, M.G. \& ANJOS, L.H.C. Aggregate distribution and soil organic matter under different tillage system for vegetable crops in a Red Latosol from Brazil. Soil Till. Res., 77:79-84, 2004. 
QUALLS, R.G.; HAINES, B.L.; SWANK, W.T \& TYLER, S.W. Retention of soluble organic nutrients by a forested ecosystem. Biogeochemistry, 61:135-171, 2002.

RESENDE, M.; CURI, N.; REZENDE, S.B. \& CORRÊA, G.F. Pedologia: Base para distinção dos ambientes. 5.ed. Lavras, Universidade Federal de Lavras, 2007. 322p.

ROSS, D.J.; TATE, K.R.; SCOTT, N.A. \& FELTHAM, C.W. Land use change: Effects on carbon, nitrogen and phosphorus pools and fluxes in three adjacents ecosystems. Soil Biol. Biochem., 31:803-813, 1999.

SCHWESIG, D.; KALBITZ, K. \& MATZNER, E. Effects of aluminium on the mineralization of dissolved organic carbon derived from forest floors. Europ. J. Soil Sci., 54:311-322, 2003.

SHEPHERD, T.G.; SAGGAR, S.; NEWMAN, R.H.; ROSS, C.W. \& DANDO, J.L. Tillage-induced changes to soil structure and organic carbon fractions in New Zealand soils. Austr. J. Soil Res., 39:465-489, 2001.

SILVA, I.R. \& MENDONÇA, E.S. Matéria orgânica do solo. In: NOVAIS, R.F.; ALVAREZ V., V.H.; BARROS, N.F.; FONTES, R.L.F.; CANTARUTTI, R.B. \& NEVES, J.C.L., eds. Fertilidade do solo. Viçosa, MG, Sociedade Brasileira Ciência do Solo, 2007. p.275-374.
SMOLANDER, A. \& KITUNEN, V. Soil microbial activities and characteristics of dissolved organic $\mathrm{C}$ and $\mathrm{N}$ in relation to tree species. Soil Biol. Biochem., 34:651-660, 2002.

TAPIA-CORAL, S.C.; LUIZÃO, F.J.; WANDELLI, E.L. \& FERNANDES, E.C.M. Carbon and nutrient stocks in the litter layer of agroforestry systems in central Amazonia, Brazil. Agrofor. Syst., 65:33-42, 2005.

USSIRI, D.A.N. \& JOHNSON, C.E. Sorption of organic carbon fractions by spodosol mineral horizons. Soil Sci. Soc. Am. J., 68:253-262, 2004.

WATERLOO, M.J.; OLIVEIRA, S.M.; DRUCKER, D.P.; NOBRE, A.D.; CUARTAS, L.A.; HODNETT, M.G.; LANGEDIJK, I.; JANS, W.W.P.; TOMASELLA, J.; ARAUJO, A.C.; PIMENTEL, T.P. \& ESTRADA, J.C.M. Export of organic carbon in run-off from an Amazonian rainforest blackwater catchment. Hydrol. Proc., 20:2581-2597, 2006. 